\title{
How Effective is Online Couple Relationship Education? A Systematic Meta-Content Review
}

\author{
Allison Megale ${ }^{1}\left[\right.$ [D $\cdot$ Emily Peterson ${ }^{1} \cdot$ Myrna L. Friedlander $^{1}$ \\ Accepted: 9 May 2021 / Published online: 17 May 2021 \\ (c) The Author(s), under exclusive licence to Springer Science+Business Media, LLC, part of Springer Nature 2021
}

\begin{abstract}
Due to logistical and financial barriers that keep many distressed couples from seeking psychotherapy, online relationship education is a more accessible alternative. In the decade since a web-based program showed equivalent effectiveness to traditional marriage education (Duncan et al., 2009), several fully online programs have been developed and evaluated. We reviewed nine studies of four different programs that sampled 2,000 + couples. Specifically, we rated each study's experimental rigor and compared research designs, theoretical and empirical grounding, average post-intervention and follow-up effect sizes, and differential effectiveness. Across studies, measured outcomes included relational (improved satisfaction, quality, confidence, commitment, communication; reduced conflict and aggression) and individual functioning on various indices of mental and physical health, emotional expression, and quality of life. Finally, we discuss the strengths and limitations of the research evidence, describe the two most evidence-based programs (ePREP and OurRelationship) in some detail and make recommendations for future study of these promising kinds of interventions.
\end{abstract}

Keywords Couple relationship education · Online couple interventions · Marriage education $\cdot$ EPREP · OurRelationship

Despite widespread acknowledgement that relationship problems are a primary source of individuals' mental and behavioral health problems (e.g. Whisman \& Uebelacker, 2003), less than a third of distressed couples seek conjoint therapy (Cicila et al., 2014). It has long been recognized that an array of logistic barriers discourage many couples from seeking professional help, including cost, transportation, and scheduling difficulties (McAllister et al., 2012).

Recognizing these barriers and the personal, social, and economic costs of family dissolution, many marital education, enrichment and divorce prevention programs have been developed, the earliest of which were systematically reviewed by Giblin et al. (1985). Generally speaking, the aim of these psychoeducational programs is to teach couples

Allison Megale

amegale@albany.edu

Emily Peterson

epeterson@albany.edu

Myrna L. Friedlander

mfriedlander@albany.edu

1 Department of Educational \& Counseling Psychology, University At Albany, State University of New York, 1400 Washington Ave, Albany, NY 12222, USA about healthy romantic relationships, effective problem solving, and conflict management (Markman \& Rhoades, 2012). Several recent content reviews and meta-analyses have shown that in-person relationship education, as well as self-directed programs with little or no professional involvement, can enhance couples' communication and improve the long-term quality of their relationships (e.g. Hawkins et al., 2010; Hawkins \& Erickson, 2015; Jakubowski et al., 2004).

A decade ago, Halford and Casey (2010) proposed using web-based technology to "enhance the reach and impact" of relationship education, noting that teaching couples evidence-based communication skills has the potential not only to reduce relational conflict, aggression, and separation but also to improve individuals' personal and work functioning (p. 111). The increased availability of the internet in recent years (Anderson, 2019), along with surveys indicating that many people prefer structured web-based help to psychotherapy (Georgia \& Doss, 2013), prompted the rapid development of several online relationship programs for couples. Over the past decade, these programs have provided a readily accessible source of scientifically based help for thousands of couples. As a private, lower-cost alternative, couples who enroll in online relationship education programs can work at 
their own pace when it is convenient for them and without needing to leave home or arrange for childcare.

According to a recent survey (Roddy et al., 2019), the top five relationship problems endorsed by couples seeking online help are difficulties with communication and emotional intimacy, spouse-specific issues (e.g. addictive behaviors), issues with trust, and frequent arguments. Although not all of these problems are specifically addressed in every online intervention, internet-based programs typically have a skills-based component that teaches couples how to communicate effectively, resolve conflict and solve problems (Cicilia et al., 2014). While the various online programs vary in content, length, use of homework and paraprofessional coaching, generally they include some combination of readings and videos.

The first outcome study of a "web-based marriage education" program showed equivalent effectiveness to an inperson relationship workshop (Duncan et al., 2009), both of which were grounded in Gottman's $(1994,1999)$ longitudinal studies of couple communication. Since this initial experimental trial, numerous controlled studies of online relationship programs have been published, as have surveys to identify why many couples prefer online self-help resources to in-person professional help (e.g. Georgia \& Doss, 2013; Roddy et al., 2016). Despite the growing interest in online couple relationship education (CRE), a comparative analysis of the effectiveness and characteristics of these programs is lacking.

To fill this gap, we reviewed all English language, fully online CRE programs that have been subjected to at least one experimental trial. In addition to comparing target populations, program characteristics, research designs and efficacy rates, we discuss the strengths and limitations of the research evidence, describe the two most evidence-based programs, and provide suggestions for future study. Due to the rapid growth of online CRE and its current appeal since the onset of the coronavirus pandemic, we reasoned that program developers, researchers, and referring practitioners could benefit from a synthesized comparative review.

\section{Method}

\section{Selection of Studies and Coding Procedure}

We followed PRISMA guidelines (Moher et al., 2009) to locate experimental trials of online couple relationship education (CRE) programs published through 2020. We identified an online CRE program as one that its developers described as a computer-based, web-based or online intervention or program whose aim is to enhance couples' romantic relationships. Programs termed flexible delivery or self-directed were excluded if they were not exclusively delivered online.

To begin, we located all of the experimental studies referenced in Cicila et al.'s (2014) review of combining internet-based interventions with traditional couple therapy and McAllister et al.'s (2012) meta-analytic review of selfdirected (including online) CRE programs.

We also conducted an electronic search of several databases using the search terms online couple relationship education, marriage education, online relationship prevention programs, online relationship education programs, among others. Additionally, we searched by the titles of the named online relationship programs within several databases and cross-referenced articles by their titles and abstracts in 13 clinical, systemic, and couple and family therapy journals. We contacted the principal investigators of previous studies of fully online programs to locate any unpublished or in-press research on the topic. The search process yielded a total of 23 English language articles that met our initial criteria.

From this initial pool, we excluded studies in which (a) only one member of the couple participated, (b) relational outcomes were not assessed, (c) no psychoeducation was provided, and (d) an in-person component was an integral part of the program. On the other hand, due our interest in fully evaluating online CRE, we included one comparative study of a specific program component (i.e. varying amounts of coach support in Roddy et al., 2018).

Based on these criteria, we retained 16 articles that reported on 9 prospective investigations, 3 follow-up studies, and 4 secondary analyses of the efficacy and/or effectiveness of online CRE. The 4 programs whose effectiveness was reported in these articles were (1) OurRelationship (Doss et al., 2016, 2019, 2020; Georgia Salivar, 2018, 2020; Roddy Nowlan, \& Doss, 2017; Roddy Rothman, \& Doss, 2018; Roddy et al., 2020, Roddy et al. 2020b; Rothman et al., 2019), which was adapted from Integrative Behavioral Couple Therapy (Christensen et al., 2004); (2) ePREP (Braithwaite \& Fincham, 2011, 2014; Doss et al., 2020) and PREP (Loew et al., 2012), two adaptations of Prevention and Relationship Education Preparation workshops (Stanley et al., 2006), which are based on extensive, longitudinal marital research (e.g. Markham et al., 1993); (3) an unnamed marriage education program (Duncan et al., 2009) based on Gottman's (1994, 1999) couple interaction research, a selfhelp book (Markman et al., 2001) and a clinician handbook (Stuart, 2004); and (4) a relationship excitement program (Coulter \& Malouff, 2013), which was suggested by studies on the influence of new and exciting activities on relationship quality (Aron et al., 2000).

To compare specific characteristics and efficacy rates across studies, we coded each investigation on several criteria, including sample size, type of control group, 
Table 1 Characteristics of the Controlled Efficacy Trials

\begin{tabular}{|c|c|c|c|c|c|c|c|c|c|c|}
\hline \multirow[t]{2}{*}{ Variable } & \multicolumn{4}{|c|}{ OurRelationship } & \multicolumn{3}{|c|}{ (e)PREP } & \multirow{2}{*}{$\begin{array}{l}\text { Our Relation- } \\
\text { ship vs. ePREP }\end{array}$} & \multirow{2}{*}{$\begin{array}{l}\text { Marriage } \\
\text { Education }\end{array}$} & \multirow{2}{*}{$\begin{array}{l}\text { Relation- } \\
\text { ship Excite- } \\
\text { ment }\end{array}$} \\
\hline & 1 & 2 & 3 & 4 & 5 & 6 & 7 & & & \\
\hline Type of control group & wl & $\mathrm{c}$ & c & $\mathrm{c}$ & ap & ap & ap & wl & $\mathrm{c}$ & wl \\
\hline \# of couples & 300 & 104 & 356 & 885 & 77 & 32 & 52 & 742 & 82 & 101 \\
\hline Compensation to couples & $\mathrm{x}$ & $\mathrm{x}$ & - & - & - & $\mathrm{x}$ & $\mathrm{x}$ & $\mathrm{x}$ & $\mathrm{x}$ & - \\
\hline Program length (weeks) & 8 & $6^{\mathrm{a}}$ & 6 & 9 & 6 & 1 & 1 & 6 & 6 & 4 \\
\hline Coach included & $\mathrm{x}$ & $x^{b}$ & $\mathrm{x}$ & $\mathrm{x}$ & - & - & - & $\mathrm{x}$ & $\mathrm{x}$ & - \\
\hline Homework required & - & - & - & - & $\mathrm{x}$ & - & - & $\mathrm{x}$ & $\mathrm{x}$ & - \\
\hline Follow-up assessment & $\mathrm{x}$ & $\mathrm{x}$ & - & - & $\mathrm{x}$ & - & $\mathrm{x}$ & $\mathrm{x}$ & $\mathrm{x}$ & $\mathrm{x}$ \\
\hline Measure of relational distress & $\mathrm{x}$ & $\mathrm{x}$ & $\mathrm{x}$ & $\mathrm{x}$ & - & - & - & $\mathrm{x}$ & - & - \\
\hline Individual outcome measures & $\mathrm{x}$ & $\mathrm{x}$ & $\mathrm{x}$ & $\mathrm{x}$ & $\mathrm{x}$ & - & - & $\mathrm{x}$ & - & $\mathrm{x}$ \\
\hline \multicolumn{11}{|l|}{ Relational effect size $^{c}$} \\
\hline Pre-/Post-treatment & .58 & $.63, .54^{\mathrm{d}}$ & $52, .61^{\mathrm{e}}$ & $\mathrm{nr}$ & $\mathrm{nr}$ & .56 & .22 & $72, .60^{f}$ & .18 & .44 \\
\hline Pre-/Follow up & .10 & $.05, .28^{\mathrm{d}}$ & - & - & $\mathrm{nr}$ & - & .18 & $.07 .14^{\mathrm{f}}$ & - & .07 \\
\hline \multicolumn{11}{|l|}{ Individual effect size ${ }^{c}$} \\
\hline Pre-/Post-treatment & .47 & $.52, .41^{\mathrm{d}}$ & $.45, .65^{\mathrm{e}}$ & $\mathrm{nr}$ & $\mathrm{nr}$ & - & - & $.26^{\mathrm{f}}$ & $\mathrm{nr}$ & $\mathrm{nr}$ \\
\hline Pre-/Follow up & .08 & $.10, .09^{\mathrm{d}}$ & - & - & - & - & - & $.05, .10^{\mathrm{f}}$ & - & $\mathrm{nr}$ \\
\hline Experimental rigor & 1 & 2 & 1 & 2 & 1 & 3 & 1 & 1 & 2 & 1 \\
\hline
\end{tabular}

OR=OurRelationship. $1=$ OR (Doss et al. (2016; follow up 2019); $2=$ OR-Brief (Roddy et al., 2017); $3=$ OR (Roddy et al., 2018); $4=$ OR (Rothman et al., 2019); $5=$ ePREP (Braithwaite \& Fincham, 2011); 6=PREP (Loew et al., 2012); 7 =ePREP; (Braithwaite \& Fincham, 2014); OurRelationship vs. ePREP (Doss et al., 2020; follow up: Roddy, Rhoades, et al., 2020; Roddy, Knopp, et al., 2020);

Marriage Education $=($ Duncan et al., 2009); Relationship Excitement $=($ Coulter \& Malouff, 2013); wl $=$ wait list control; c $=$ comparative treatments (no wait-list or attention-placebo group); ap=attention placebo control

${ }^{\mathrm{a}}$ Mean number of weeks

${ }^{\mathrm{b}}$ Coaching was compared with no coaching

${ }^{\mathrm{c}}$ Relational effect $\mathrm{size}=$ absolute value mean of the within-group Cohen's $d$ s across all relational outcome measures; individual effect size $=$ absolute value mean of the within-group Cohen's $d$ s across all individual outcome measures

${ }^{\mathrm{d}} 63$ in the no coaching condition, .54 in the coaching condition. $\mathrm{nr}=$ not reported

${ }^{\mathrm{e}} 52$ in the low coaching condition, .61 in the high coaching condition

${ }^{\mathrm{f}} 72$ in the OR group, .60 in the ePREP group. Experimental rigor: $1=$ good, 2 =fair, $3=$ poor (based on the risk of bias criteria in the Cochrane Collaboration Tool; Higgins et al., 2011)

length of intervention, compensation to participants, use of coaching, types of outcome(s), average within-program effect size(s) at post-intervention and, if available, at follow up. Additionally, we rated each study for its experimental rigor using the risk of bias criteria in the Cochrane Collaboration Tool (CCT; Higgins et al., 2011). Although this tool was developed for assessing the rigor of medical trials, most of the criteria also apply to psychosocial intervention trials: random sequence generation, allocation concealment, blinding of participants, blinding of outcome assessment for participants, reporting of incomplete outcome data, and selective reporting.

The first two authors independently rated the nine experimental studies on each of the six CCT criteria as having either a low, medium, or high risk of bias. Next, using Higgins et al. (2011) recommendations for synthesizing these CCT ratings, the overall experimental rigor of each intervention trial was judged as either $1=$ good, $2=$ fair, or $3=$ poor .
In one case of disagreement, the difference was negotiated to consensus.

\section{Comparative Results}

\section{Study Characteristics and Experimental Rigor}

Table 1 summarizes various characteristics of the nine experimental studies of CRE. The two most researched programs, OurRelationship (OR; two controlled and two uncontrolled trials; Doss et al., 2016; Roddy et al., 2017, 2018) and PREP/ePREP (three controlled trials; Braithwaite \& Fincham, 2011, 2014; Loew et al., 2012), were recently compared by Doss et al. (2020) The programs ranged from exceptionally short, PREP (one week; Loew et al., 2012) and Brief-OR (two weeks; Roddy et al., 2017) to 8 weeks (Doss et al., 2016), with the other programs ranging from 4 
to 8 weeks. Adjunctive coaching by a professional or paraprofessional was provided in Duncan et al.'s (2009) marriage education program and in one or more conditions in all of the OR studies, including the recent comparative trial of OR versus PREP (Doss et al., 2020).

Across studies, sample sizes ranged from 32 (PREP; Loew et al., 2012) to 885 (OR; Rothman et al., 2019) couples, with notably larger samples in the OR studies. In the first comparative online study, $98.8 \%$ of participants belonged to the Church of Jesus Christ of Latter-day Saints; most were college-educated couples in their first marriage who did not report significant relationship distress and who, by virtue of their religion, were unlikely to divorce (Duncan et al., 2009). In the first OR study, Doss et al. (2016) described the sample as representative of the U.S. in terms of racial, ethnic and educational background, although sexual minority couples were excluded as were couples in shortterm relationships and those with severe individual and relationship problems. In the OR samples, one or both members of the couple reported at least a moderate level of relational distress (Doss et al., 2016, 2020; Roddy et al., 2017, 2018); one study specifically sampled low-income couples (Doss et al., 2020). In the first ePREP study, Braithwaite and Fincham (2011) sampled mostly white college students in committed dating relationships; in the subsequent ePREP study (Braithwaite \& Fincham, 2014), the community sample of couples, married for $M=4.3$ years, was somewhat more heterogeneous and racially diverse, with $35 \%$ participants of Color. In the only study that tailored its content (PREP) to couples' specific life circumstances, Loew et al. (2012) sampled a high-risk group, foster and adoptive parents, most of whom were white and had been married for 15 years, on average.

In several studies, participants were recruited and compensated for completing surveys and allowing their data to be analyzed for research purposes. Compensation fees ranged from $\$ 25$ (Duncan et al., 2009) to $\$ 70$ (Braithwaite $\&$ Fincham, 2014). Doss et al. (2020) paid individuals $\$ 25$ for each completed survey (four over six months) and $\$ 15$ per person for attending each coaching call.

Six of the nine prospective studies used a wait-list (Coulter \& Malouff, 2013; Doss et al., 2016, 2020; Duncan et al., 2009) or attention placebo control that either involved reading parenting information for adoptive parents (Loew et al., 2012) or material on anxiety, depression, and relationships (Braithwaite \& Fincham, 2011, 2014). Three studies investigated OR effectiveness with differing amounts of coaching support (Roddy et al., 2017, 2018; Rothman et al., 2019), but without a no-treatment control group.

Table 1 shows that only the OR studies assessed participants' pre-intervention levels of relational distress. With two exceptions (Braithwaite \& Fincham, 2014; Loew et al., 2012), the primary outcome was improvement in couples' self-reported relationship quality or satisfaction. Other targeted relational outcomes were improvements in communication skills, both observed (Braithwaite \& Fincham, 2011) and self-reported (Duncan et al., 2009; Loew et al., 2012; Roddy et al., 2017); relationship confidence, commitment (Braithwaite \& Fincham, 2014); emotional support (Doss et al., 2020) and relationship excitement (Coulter \& Malouff, 2013). Reductions in communication conflict (Doss et al., 2020) and intimate partner aggression (Braithwaite \& Fincham, 2011, 2014; Doss et al., 2020) were also assessed in a few studies.

In addition to these relational outcomes, individual functioning was measured in all of the OR studies and one ePREP study (Braithwaite \& Fincham, 2011). Specifically, these studies targeted aspects of partners' physical (Doss et al., 2016, 2020) and mental health, particularly their selfreported anxiety and depression (Braithwaite \& Fincham, 2011; Doss et al., 2016, 2020), as well as their psychological distress, anger, stress, health, insomnia and problem alcohol use (Roddy, Rhoades, et al., 2020). Reflecting the relationship enhancement aim of some programs, three studies measured changes in partners' positive emotions (Coulter \& Malouff, 2013), work functioning, amount of exercise and/ or quality of life (Doss et al., 2016, 2020).

In terms of data analysis, all the OR studies used multilevel modeling to account for nested data (i.e. time within partners and partners within couples). The two ePREP studies used the actor-partner interdependence model to determine whether, for example, women's mastery of the online material (in terms of quiz scores) would predict their own and/or their male partners' improvements in communication skills (Braithwaite \& Fincham, 2011).

Finally, based on the procedures for assessing risk of bias described earlier, we rated six of the nine prospective studies as having "good" experimental rigor (Braithwaite et al., 2011, 2014; Coulter \& Malouff, 2013; Doss et al., 2016, 2020; Roddy et al., 2018; see Table 1). These studies included a waitlist or attention control; Roddy et al. (2018) compared OR with and without coaching. The two "fair" studies (Duncan et al., 2009; Roddy et al., 2017) either did not have a wait-list or attention-placebo control group, did not randomly assign all participants, ${ }^{1}$ did not report effect sizes for all groups, or had a high attrition rate. The only study rated "poor" (Loew et al., 2012) was described as a pilot test and had a small sample (32 couples), which raised the likelihood of Type II error.

\footnotetext{
${ }^{1}$ Roddy et al. (2017), participants recruited for Brief-OR (with or without coaching support) had been participants on the 6-month wait list in Doss et al. (2016). In Rothman et al. (2019), participants in Roddy et al. (2018) who had received high or low coaching support were contrasted with a control group that consisted of previous OR participants who had received no coaching support.
} 


\section{Efficacy, Effectiveness, and Program Evaluation}

In the six trials in which a randomly assigned experimental group was contrasted with a wait-list or attention placebo control group (see Table 1), all results significantly favored the online CRE program. To compare pre-/post-intervention and pre-/follow-up efficacy rates across the four programs, we averaged within-treatment effect sizes across the measured (a) relational and (b) individual variables. (When no within-program effect sizes were reported by the authors, we calculated them, if reported, from the published pre- and post- means and standard deviations.) In the prospective studies published since Duncan et al.'s (2009) comparison of a web-based program (mean $d=0.18$ ) to a face-to-face workshop (mean $d=0.25$ ), average within-group effect sizes were modest to substantial for the relational $(0.22$ to 0.63$)$ and individual outcomes ( 0.26 to 0.65 ; see Table 1$)$. Notably, effect sizes for the 4-week relationship excitement program (Coutler \& Malouff, 2013) (mean $d=0.44$ ) were smaller than the 2-week Brief-OR (mean $d s=0.54$ to 0.63 ; Roddy et al., 2017) and the lengthier (6- to 8-week) OR programs (Doss et al., 2016, 2020; Roddy et al., 2018), in which the mean $d$ s ranged from 0.61 to 0.72 .

In terms of post-intervention program evaluation, participants in Loew et al.'s (2012) pilot study reported being satisfied with PREP and rated its content favorably in terms of its realism, helpfulness and usefulness. Participants in OR (Doss et al., 2016; Roddy et al., 2018) and ePREP (Doss et al., 2020) were also satisfied and had moderate to high rates of program completion (Doss et al., 2020; Roddy et al., 2018).

Follow-up assessments varied in timing and had average pre- to follow-up effect sizes ranging from $d=0.05$ to 0.28 . Studies with a single follow-up point re-assessed program participants' outcomes at either 6 weeks (Braithwaite \& Fincham, 2011), 3 months (Duncan et al., 2009; Roddy et al., 2017), 4 months (Coulter \& Malouff, 2013), or 12 months (Braithwaite \& Fincham, 2014). The randomized controlled trials of OR (Doss et al., 2016) and OR vs. ePREP (Doss et al., 2020) had multiple follow-up assessments ranging from 2 to 12 months (Doss et al., 2019, 2020; Roddy, Knopp, et al., 2020; Roddy, Rhoades, et al., 2020).

Three of the OR studies tested the specific effectiveness of coaching by paraprofessionals or psychology graduate students. In the first study, coaching was included as an integral part of the program (Doss et al., 2016). Subsequently, Roddy et al. (2017) found that compared to participants who only received automated email contacts, Brief-OR participants who received two supportive coaching calls had higher rates of completion $(71.2 \%$ versus $42.3 \%$ ), although the groups' average effect sizes did not differ substantially. In later comparations of low versus high coaching support, defined as one versus four coaching contacts, Roddy et al. (2018) found that while both groups reported significant changes in relationship satisfaction, anxiety and depression, participants who received more coaching support experienced a greater reduction in anxiety. Notably, Rothman et al. (2019) found higher rates of program completion among Roddy et al.'s (2018) participants who received four 15 -min coaching calls $(66.1 \%)$ compared to participants who received a single coaching call $(36 \%)$ or no coaching at all $(6.1 \%)$.

In the recent, comparative trial of OR, ePREP and waitlist controls (with $N=752$ low-income couples), most participants $(81 \%)$ were relationally distressed (Doss et al., 2020). At post-intervention, both programs demonstrated substantially increased relationship satisfaction and emotional support, as well as reduced communication conflict and intimate partner violence. Moreover, at the 6-month follow up the two programs showed almost identical rates of clinically significant recovery in terms of relationship satisfaction (29\%) compared to controls (12\%). Across outcome variables, the averaged pre-/post- effect sizes were moderate, mean $d s=0.72(\mathrm{OR})$ and 0.60 (ePREP), with one difference: The OR participants reported a significantly greater decrease in communication conflict. Doss et al. (2020) attributed this finding to the two programs' differing objectives: Whereas ePREP is a preventative program focused on basic skill building, OR helps couples solve a specific problem in their relationship using acceptance strategies.

Additionally, Doss et al. (2020) found that changes in individual functioning (in terms of psychological distress, stress, anger, problem alcohol use, health, insomnia, exercise) also showed substantive but more modest effects which did not differ by program but that exceeded the improvements of controls at posttest and follow-up (Roddy, Knopp, et al., 2020; Roddy, Rhoades, et al., 2020). Notably, some participants continued to improve at the one-year follow up (Roddy, Knopp, et al., 2020).

Finally, in terms of comparative long-term effectiveness, neither OR nor ePREP reduced the rate of relationship dissolution more so than controls at posttest or the 6-month follow up (Doss et al., 2020). On the other hand, all aspects of relationship functioning other than IPV, including thoughts of breaking up, were maintained at the 4-, 6-, and 12-month follow ups (Roddy, Knopp, et al., 2020). Notably, at the 12-month follow up relational outcomes slightly favored ePREP (mean $d=0.14$ ) over OR (pre- to follow-up mean $d=0.07$ ) (Roddy, Knopp, et al., 2020). A similar pattern emerged for the individual outcomes (Roddy, Rhoades, et al., 2020), pre- to follow-up mean $d \mathrm{~s}=0.10$ (ePREP) versus $0.05(\mathrm{OR})$. 


\section{Moderators: Who Benefits Most?}

With respect to differential effectiveness, the first ePREP study (Braithwaite \& Fincham, 2011) with college students found stronger effects among couples who began the program with relatively poorer communication skills and higher levels of psychological aggression. The authors concluded that ePREP may be less beneficial for couples who are already skilled at conflict resolution. In the second ePREP study, reduced intimate partner aggression at postintervention and a one-year follow up was reported by both men and women (Braithwaite \& Fincham, 2014). Although both studies took gender into account by using the actorpartner interdependence model, the few gender differences that emerged were not discussed in depth.

Examining Doss et al.'s (2016) first OR study by participants' demographic characteristics, Georgia Salivar et al. (2018) found largely similar outcomes among traditionally underserved couples (members of racial/ethnic minority groups, lower income and rural couples) and non-underserved couples, although rural couples reported fewer positive relationship qualities and Hispanic couples reported more posttest improvement in perceived health but less improvement in relationship confidence. Low-income and Hispanic couples were more likely to drop out of the program despite program evaluation rates similar to those reported by the higher income and non-Hispanic couples (Georgia Salivar et al., 2018). Follow-up assessments (Doss et al., 2019) showed that the underserved couples reported maintaining their post-intervention gains at the same rate as the traditionally served couples; at the 12-month assessment point, Hispanic couples reported continued improvements in relationship quality and confidence.

In Roddy et al.'s (2018) OR study, neither race nor household income moderated the effectiveness of OR delivered with high or low coaching support. In comparing participants from Roddy et al.'s (2018) high and low coaching groups to a no-coach condition, Rothman et al. (2019) found that coaching support in OR seemed to be most helpful for Hispanic participants and those who did not begin the program with high levels of depression.

Finally, the recent comparative OR/ePREP trial (Doss et al., 2020) found no differences in effectiveness by participants' initial levels of relationship distress. Among the mostly low-income participants in the sample, those with more mental and physical health problems reported the most gains post-intervention and at the four-month (Roddy, Rhoades, et al., 2020) and one-year (Roddy, Knopp, et al., 2020) follow-up assessments. In a secondary analysis of Doss et al. (2020) that compared 90 military couples (those with at least one active duty or veteran partner) to 642 civilian couples, Georgia Salivar et al. (2020) found similar rates of program satisfaction but lower completion rates in the military group. Although both groups showed comparable improvements at post-treatment and at follow-up on the relationship measures, the military couples made fewer gains on measures of individual functioning and intimate partner violence.

\section{Description of OurRelationship: https:// www.ourrelationship.com/}

The most thoroughly investigated of the four online programs, OurRelationship is the only CRE based on an evidence-based approach to couple therapy, Integrative Behavioral Couple Therapy (IBCT; Christensen et al., 2004; Jacobson \& Christensen, 1996; Jacobson et al., 2000;). Due to its adaptation from psychotherapy, OR is specifically designed to be an intervention for relationship distress. Currently offered as a flexible, self-paced, one-to-two month program, the objective is to assist couples identify and work through a specific problem in their relationship before the issue is serious enough to require professional help. Similar to IBCT, OR uses acceptance-based strategies, such as understanding individual differences, empathic joining and unified detachment, to help couples resolve a problem of their choosing. Throughout the program, couples engage in online activities and review content that include "slides with text, animations, videos, journaling, and questions in text, multiple choice, and drop-down format" (Roddy et al., 2017, p. 839). Partners complete most activities independently; after each phase, they are directed to have structured conversations with one another about their responses.

Three program phases are organized around the acronym OUR: "first $O$ bserving the problem through objective feedback, subsequently Understanding the problem through a detailed, objective analysis of the problem, and then beginning to Resolve the problem through efforts to enact specific behavioral changes" (Doss et al., 2013, p. 143). In the Observing phase, individuals are given feedback regarding salient issues in their relationship in order to identify which specific issue should be addressed during the program. In the Understanding phase, couples work through the content separately in order to develop a DEEP understanding of the couple's identified problem. DEEP refers to "the role that natural differences, surface and hidden emotions, external stress, and patterns of communication have played in the development and exacerbation of their core issue" (Doss et al., 2013, p. 143). At the end of the Understanding phase, partners share what they have learned based on a more indepth understanding of the identified problem. Lastly, in the Respond phase, psychoeducational material is presented about acceptance and communication for couples to use in creating a plan about individual changes that can be made 
in order to improve the couple's identified issue. Feedback tailored to each couple is offered at the conclusion of the program, with referrals if needed.

The OR program is designed for members of a couple to work through the structured material at the same pace (typically for seven hours over six weeks). Doss et al. (2013) noted that this programming allows for flexible completion and allows partners to reflect deeply on their relational concerns. Reflecting the evidence that high coaching support is an effective component of OR (see above), the program comes with the option of four 20-min telehealth coaching calls. Although to date no studies have evaluated OR for same-gender couples, the website offers a version of the program specifically for this population. In 2020, the cost was $\$ 50$ for the self-guided option and $\$ 150$ for the coaching option.

\section{Description of ePREP: https://www.lovet akeslearning.com/index.php}

The second most researched program, ePREP, evolved from PREP workshops (e.g. Markman et al., 2001) that are based on marital research conducted over a 40-year period (e.g. Stanley et al., 1999). The original PREP program was offered in two formats, as weekly sessions with four to eight couples or as a condensed, three-day workshop with 20 to 40 couples.

Unlike OR, which can be considered tertiary prevention since it targets couples' ongoing conflicts (Renick et al., 1992), ePREP, like its forerunner PREP, is considered primary prevention (Braithwaite \& Fincham, 2007) since it targets communication difficulties before they detract from a couple's relationship satisfaction or commitment. The assumption is that if couples are able to develop healthy communication patterns before their conflicts become problematic, they will be better equipped to handle conflictual issues that arise in the future.

ePREP was originally tested with individuals (Braithwaite \& Fincham, 2007). Results showed that some of the posttest gains attenuated at a 10-month follow-up (Braithwaite \& Fincham, 2007). The subsequently developed modification for couples (Braithwaite \& Fincham, 2011) showed more "immediate and robust" results (Braithwaite \& Fincham, 2014, p. 13).

ePREP consists of narrated modules with psychoeducational material, videos of couple interactions and specific questions for couples to answer (Braithwaite \& Fincham, 2014). The seven modules are "(1) Background on risk factors for relationship problems: communication danger signs, (2) Description of how communication filters can impaire constructive communication, (3) Description of how normal patterns of communicating fail to address deeper issues and why addressing these deeper issues is a key element of having a healthy relationship, (4) Communication skills training, (5) Problem solving training, (6) Enhancing fun and friendship, and (7) Final section reviewing techniques and setting up plan to complete weekly homework assignments" (p. 14). ePREP techniques include the Time Out Ground Rule, XYZ Communication, the Speaker-Listener Technique, and the "Fun Deck" Technique.

The ePREP website does not describe the program as one that romantic partners to complete together, although a brief video depicts a couple arguing before and after learning ePREP communication skills. Rather, the "self-paced" ePREP program is advertised to as a way for individuals or partners in dating or committed relationships to "protect [their] love life" by helping them increase their "dedication and commitment," even if they are not struggling with their relationship. In 2020, the cost was $\$ 34.95$ for the six-hour program which remains available to users for a six-month period.

\section{Discussion}

In contrast to the many previous reviews and meta-analyses of traditional relationship, the present review was limited to fully online programs published through 2020 . We located nine prospective studies of four different online programs. As a group, these randomized trials sampled $2000+$ couples, ${ }^{2}$ establishing the efficacy of online CRE for improving (a) participants' self-reported relationship satisfaction, commitment, confidence, communication skills, and (b) to a more limited extent, individuals' emotional/behavioral problems, e.g. anxiety, depression, anger, stress, aggression, and problem alcohol use. Moreover, several programs have shown positive effects on participants' emotional expression, quality of life, and work functioning.

Aside from the first published study of online CRE (Duncan et al., 2009), investigations of the three other programs demonstrated medium within-program effect sizes, on average, at posttest, with more modest effects at follow up. These efficacy rates, even those for the two very brief programs (Loew et al., 2012; Roddy et al., 2017), compare favorably to the effect sizes reported in two meta-analyses of traditional CRE (Hawkins et al., 2008; Hawkins \& Erickson, 2015) and, in the case of OR, to its psychotherapy counterpart, IBCT (Georgia Salivar et al., 2020). Additionally, participants in both ePREP and OR indicated high rates of satisfaction.

Moreover, a lack of significant differences between OR and ePREP on most outcomes (Doss et al., 2020), along

\footnotetext{
${ }^{2}$ In some of the OR studies, the samples overlapped, so that an exact count was not possible.
} 
with their comparable efficacy rates, is notable since the two programs were developed independently, target relational functioning differently, and have many different program features. From its inception, ePREP evolved from a preventative, evidence-based behavioral intervention designed to enrich relationships by teaching couples effective skills to manage conflict (e.g. Markman et al., 2001). In contrast, OurRelationship was created to help moderately distressed couples using the active elements in IBCT, which aims to change behavior through acceptance (Doss et al., 2016). Due to its adaptation from psychotherapy to relationship education, only OR targets partners' relationship distress as well as their level of satisfaction.

Despite these historical and conceptual differences, post-intervention and follow-up data indicate that both the behavior-focused ePREP and the acceptance-focused OR can effectively address the kinds of relationship dynamics that distressed couples seeking online help identify as most troublesome (Roddy et al., 2019). Additionally, participation in either program can potentially reduce depression (Braithwaite \& Fincham, 2011; Doss et al., 2016; Roddy et al., 2017, 2018), which often co-occurs with relationship distress (Gupta et al., 2003).

Our analysis of the studies' risk of bias (Higgins et al., 2011) indicated "good" experimental rigor in 6 of the 9 investigations. In reviewing these studies, we noted several other strengths. All four programs followed a specific sequencing of written material, and while the relationship excitement program (Coulter \& Malouff, 2013) is atheoretical in content, the other three programs have solid theoretical and empirical grounding. In terms of reporting, several studies provided flow charts (from participant recruitment to randomization, follow up, and analysis) and assessed group differences in participants' characteristics. The ePREP studies used active placebo controls with "inert" but relevant relational content (Braithwaite \& Fincham, 2011, p. 127), a strong design feature in experimental trials. To account for the lack of independence in data provided by two members of a couple, all the OR studies used multilevel modeling, and the two ePREP studies used the actor-partner interdependence model.

On the other hand, generalizability of the demonstrated results, while impressive for a single decade of experimentally controlled investigations, is nonetheless limited. The samples in the two ePREP studies were much smaller and more homogeneous than those in the OR trials, which had larger, more racially and ethnically diverse samples. Only two studies (Coulter \& Malouff, 2013; Doss et al., 2020) included sexual minority couples, and no study exclusively sampled these couples. While some investigators conducted moderator analyses to compare outcomes by gender, the lack of consistent results across studies indicates a need for replication.

A few other limitations are noteworthy. Large attrition rates occurred in some studies (Duncan et al., 2009; Rothman et al., 2019), and the wait-list controls were not always followed up (e.g. Coutler \& Malouff, 2013), limiting conclusions about the programs' long-term effectiveness. Moreover, self-selection bias can come into play when participants are recruited and financially compensated. Finally, despite consistent findings of improved relationship satisfaction in the prospective OR studies, all these trials were conducted by the same team of investigators, requiring replication by others in order to more definitively establish the program's efficacy. On the other hand, to mitigate investigator bias, the large-scale comparative study was designed and conducted by OR developers but analyzed and monitored by ePREP personnel, and the ePREP intervention was supervised by a leading ePREP researcher (Doss et al., 2020).

These limitations suggest a number of directions for additional study. Coulter and Malouff's (2013) relationship excitement intervention, although much more limited in scope than the other programs, would benefit from replication with a more diverse sample, as would Duncan et al.'s (2009) marital education program. All four online programs require longer-term follow ups to evaluate their effectiveness in preventing relationship dissolution. Aside from Duncan et al., none of the other programs have been contrasted with either face-to-face CRE workshops or traditional couple therapy. Comparative studies of (a) ePREP to PREP workshops and (b) OR to IBCT therapy could evaluate the distinct effectiveness of online CRE. Further, while some investigators assessed participants' self-reported communication skills, only one ePREP study (Braithwaire \& Fincham, 2011) involved an observation of couples' interactions. Using currently available web-based technologies, researchers could video record participants' attempts to resolve a conflict before and after participating in an online CRE intervention.

Other suggestions for future study include testing the unique contribution of active elements in established programs, such as those described by Braithwaite and Fincham (2014). Future researchers could also evaluate the inclusion of a well-established computerized inventory for couples, such as FOCCUS, PREPARE or RELATE (Halford \& Casey, 2010), for its adjunctive value. In a study that compared RELATE to RELATE + a DVD-based self-guided CRE program (Couple Care), Halford et al. (2017) found a notably stronger overall effect among participants with low initial relationship satisfaction who received the combined intervention. Future studies could use couples' RELATE results to tailor online interventions to specific profiles of relationship problems. Additionally, since we excluded studies in which only one member of the couple was sampled, 
future researchers might compare relational outcomes when one versus both partners complete the online program.

In addition to studying the active components of online CRE, researchers could evaluate the process of change over the course of the program using, for example, daily diaries or thought listing. Pre-intervention factors, such as participants' attachment style, optimism, and outcome expectations (Friedlander et al., 2019), could be tested as moderators of online CRE outcomes. The therapeutic alliance, shown to predict the effectiveness of online psychotherapy (e.g. Sucala et al., 2012), could also be studied to provide a better understanding of the well-established coaching effect in online CRE. Finally, to disentangle individual from relational outcomes, each kind of outcome could be tested as a mediator of the other kind of outcome.

All of the prospective studies in this review used selfreport data completed by individual members of a couple, and several of the acknowledged the problem of using nonindependent parametric data to understand relational outcomes. In future studies, more systemic statistical models could be used to provide an understanding of change at the couple level. As one example, the common fate model (Ledermann \& Kenny, 2012), which is estimated through structural equation modeling combined with the actor-partner interdependence model (APIM), has advantages over APIM alone in that it does not require members of a dyad to be distinguishable in some way (typically by gender). Use of this statistical approach would allow for actor/partner comparisons with same-gender couples. As another example, polynomial regression with response surface analysis (cf. Marmarosh \& Kivlighan, 2012) would allow for the study of how congruent versus discrepant predictors reported by the two members of a couple, such as initial level of distress, are associated with a criterion variable, such as post-intervention change in relationship satisfaction or commitment.

In terms of effectiveness, only the recent ePREP/OR comparative trial (Doss et al., 2020) studied participants' thoughts of break up or the actual dissolution of their relationships. Since no program differences were found at posttest or follow up (or in comparisons of CRE participants to wait-list controls), this important index of effectiveness deserves future empirical attention, especially in comparisons of online CRE with traditional couple therapy. Additionally, we concur with Halford and Casey's (2010) recommendation to expand the reach of online CRE to specific high-risk groups, now that the efficacy of the two currently available programs, OR and ePREP, has been firmly established. Indeed, the privacy of online CRE may be particularly attractive for couples with chronic conditions, including addiction and severe mental disorders; couples coping with the challenges of parenting; couples experiencing intimate partner violence or other stigmatizing circumstances, such as incarceration or removal of children; as well as any couples whose dysfunctional communication styles have been shown to predict marital dissolution (Gottman, 1999).

Finally, we encourage the developers of tested and untested traditional CRE to consider adapting their program material for online use and then evaluating its effectiveness. It is our hope that as the efficacy and value of online CRE become more widely recognized, practitioners will recommend these interventions to couples with limited financial resources and to those who are reluctant to engage in traditional psychotherapy.

\section{Declarations}

Conflict of interest The authors declare that they have no conflict of interest.

Informed Consent This manuscript does not contain any studies with human participants performed by any of the authors. As a result, informed consent was not obtained by the authors of this study since the manuscript is a review.

\section{References}

Anderson, M. (2019). Mobile technology and home broadband 2019. Pew Research Center. https://www.pewresearch.org/internet/2019/ 06/13/mobile-technology-and-home-broadband-2019/

Aron, A., Norman, C. C., Aron, E. N., McKenna, C., \& Heyman, R. (2000). Couple's shared participation in novel and arousing activities and experienced relationship quality. Journal of Personality and Social Psychology, 78, 273-283.

Braithwaite, S. R., \& Fincham, F. D. (2007). ePREP: Computer based prevention of relationship dysfunction, depression and anxiety. Journal of Social and Clinical Psychology, 26(5), 609-622.

Christensen, A., Atkins, D. C., Berns, S., Wheeler, J., Baucom, D. H., \& Simpson, L. E. (2004). Traditional versus integrative behavioral couple therapy for significantly and chronically distressed married couples. Journal of Consulting and Clinical Psychology, 72(2), 176-191.

Cicila, L. N., Georgia, E. J., \& Doss, B. D. (2014). Incorporating Internet-based interventions into couple therapy: Available resources and recommended uses. Australian and New Zealand Journal of Family Therapy, 35(4), 414-430.

Doss, B. D., Benson, L. A., Georgia, E. J., \& Christensen, A. (2013). Translation of Integrative Behavioral Couple Therapy to a webbased intervention. Family Process, 52(1), 139-153.

Friedlander, M. L., Muetzelfeld, H., Re, S., Colvin, K. F., Quinn-Nilas, C., \& Smoliak, O. (2019). Introducing the Expectations and Preference Scales for Couple Therapy (EPSCT): development psychometric evaluation, and suggested use in practice and research. Family Process, 58(4), 855-872. https://doi.org/10.1111/famp. 12446

Georgia, E. J., \& Doss, B. D. (2013). Web-based couple interventions: Do they have a future? Journal of Couple \& Relationship Therapy, 12(2), 168-185.

Georgia Salivar, E. J., Roddy, M. K., Nowlan, K. M., \& Doss, B. (2018). Effectiveness of the Online OurRelationship program for underserved couples. Couple and Family Psychology: Research and Practice, 7(3-4), 212-226. 
Georgia Salivar, E. J., Rothman, K., Roddy, M. K., \& Doss, B. D. (2020). Relative cost effectiveness of in-person and internet interventions for relationship distress. Family Process, 59(1), 66-80.

Giblin, P., Sprenkle, D. H., \& Sheehan, R. (1985). Enrichment outcome research: A meta-analysis of premarital, marital and family interventions. Journal of Marital and Family Therapy, 11(3), 257-271.

Gottman, J. (1994). Why marriages fail. The Family Therapy Networker, 18(3), 40-48.

Gottman, J. M. (1999). The marriage clinic: A scientifically-based marital therapy. WW Norton.

Gupta, M., Coyne, J. C., \& Beach, S. R. (2003). Couples treatment for major depression: Critique of the literature and suggestions for some different directions. Journal of Family Therapy, 25(4), $317-346$.

Halford, W. K., \& Casey, L. M., et al. (2010). Taking it to the people: Using technology to enhance the impact of couple relationship education. In K. Hahlweg (Ed.), Enhancing couples: The shape of couple therapy to come. (pp. 111-127). London: Hogrefe Publishing.

Halford, W. K., Rahimullah, R. H., Wilson, K. L., Occhipinti, S., Busby, D. M., \& Larson, J. (2017). Four year effects of couple relationship education on low and high satisfaction couples: A randomized clinical trial. Journal of Consulting and Clinical Psychology, 85(5), 495-507.

Hawkins, A. J., \& Erickson, S. E. (2015). Is couple and relationship education effective for lower income participants? A meta-analytic study. Journal of Family Psychology, 29, 59-68.

Hawkins, A. J., \& Fackrell, T. A. (2010). Does relationship and marriage education for lower-income couples work? A meta-analytic study of emerging research. Journal of Couple \& Relationship Therapy, 9(2), 181-191.

Hawkins, A. J., Blanchard, V. L., Baldwin, S. A., \& Fawcett, E. B. (2008). Does marriage and relationship education work? A metaanalytic study. Journal of Consulting and Clinical Psychology, 76(5), 723-734. https://doi.org/10.1037/a0012584

Higgins, J. P., Altman, D. G., Gøtzsche, P. C., Jüni, P., Moher, D., Oxman, A. D., \& Sterne, J. A. (2011). The Cochrane Collaboration's tool for assessing risk of bias in randomised trials. $B M J$, 343, d5928.

Jacobson, N. S., \& Christensen, A. (1996). Integrative couple therapy: Promoting acceptance and change. WW Norton \& Co.

Jacobson, N. S., Christensen, A., Prince, S. E., Cordova, J., \& Eldridge, K. (2000). Integrative behavioral couple therapy: an acceptancebased, promising new treatment for couple discord. Journal of Consulting and Clinical Psychology, 68(2), 351-355.

Jakubowski, S. F., Milne, E. P., Brunner, H., \& Miller, R. B. (2004). A review of empirically supported marital enrichment programs. Family Relations, 53(5), 528-536.

Ledermann, T., \& Kenny, D. A. (2012). The common fate model for dyadic data: Variations of a theoretically important but underutilized model. Journal of Family Psychology, 26(1), 140-148.

Markman, H. J., \& Rhoades, G. K. (2012). Relationship education research: Current status and future directions. Journal of Marital and Family Therapy, 38(1), 169-200.

Markman, H. J., Stanley, S. M., \& Blumberg, S. L. (2001). Fighting for your marriage: Positive steps for preventing divorce and preserving a lasting love. Jossey-Bass.

Marmarosh, C. L., \& Kivlighan, D. M., Jr. (2012). Relationships among client and counselor agreement about the working alliance, session evaluations, and change in client symptoms using response surface analysis. Journal of Counseling Psychology, 59, 352-367.

McAllister, S., Duncan, S. F., \& Hawkins, A. J. (2012). Examining the early evidence for self-directed marriage and relationship education: A meta-analytic study. Family Relations, 61(5), 742-755.
Moher, D., Liberati, A., Tetzlaff, J., Altman, D. G., \& The PRISMA Group. (2009). Preferred reporting items for systematic reviews and meta-analyses: The PRISMA statement. PLoS Med, 6(7), e1000097.

Roddy, M. K., Nowlan, K. M., Doss, B. D., \& Christensen, A. (2016). Integrative behavioral couple therapy: Theoretical background, empirical research, and dissemination. Family Process, 55(3), $408-422$.

Roddy, M. K., Rothman, K., Cicila, L. N., \& Doss, B. D. (2019). Why do couples seek relationship help online? Description and comparison to in-person interventions. Journal of Marital and Family Therapy, 45(3), 369-379.

Rothman, K., Roddy, M. K., \& Doss, B. D. (2019). Completion of a stand-alone versus coach-supported trial of a web-based program for distressed relationships. Family Relations, 68(4), 375-389.

Stanley, S. M., Amato, P. R., Johnson, C. A., \& Markman, H. J. (2006). Premarital education, marital quality, and marital stability: Findings from a large, random household survey. Journal of Family Psychology, 20(1), 117-126.

Stuart, R. B. (2004). Helping couples change. Guilford Press.

Sucala, M., Schnur, J. B., Constantino, M. J., Miller, S. J., Brackman, E. H., \& Montgomery, G. H. (2012). The therapeutic relationship in e-therapy for mental health: A systematic review. Journal of Medical Internet Research, 14(4), e110.

Whisman, M. A., \& Uebelacker, L. A. (2003). Comorbidity of relationship distress and mental and physical health problems. In D. K. Snyder \& M. A. Whisman (Eds.), Treating difficult couples: Helping clients with coexisting mental and relationship disorders. (pp. 3-26). Guilford Press.

\section{The Nine Prospective Studies in the Present Review}

Braithwaite, S. R., \& Fincham, F. D. (2011). Computer-based dissemination: A randomized clinical trial of ePREP using the actor partner interdependence model. Behaviour Research and Therapy, 49(2), 126-131.

Braithwaite, S. R., \& Fincham, F. D. (2014). Computer-based prevention of intimate partner violence in marriage. Behaviour Research and Therapy, 54, 12-21.

Coulter, K., \& Malouff, J. M. (2013). Effects of an intervention designed to enhance romantic relationship excitement: A randomized-control trial. Couple and Family Psychology: Research and Practice, 2(1), 34-44.

Doss, B. D., Cicila, L. N., Georgia, E. J., Roddy, M. K., Nowlan, K. M., Benson, L. A., \& Christensen, A. (2016). A randomized controlled trial of the web-based OurRelationship program: Effects on relationship and individual functioning. Journal of Consulting and Clinical Psychology, 84(4), 285-296.

Doss, B. D., Knopp, K., Roddy, M. K., Rothman, K., Hatch, S. G., \& Rhoades, G. K. (2020). Online programs improve relationship functioning for distressed low-income couples: Results from a nationwide randomized controlled trial. Journal of Consulting and Clinical Psychology, 88(4), 283-294.

Doss, B. D., Roddy, M. K., Nowlan, K. M., Rothman, K., \& Christensen, A. (2019). Maintenance of gains in relationship and individual functioning following the online OurRelationship program. Behavior Therapy, 50(1), 73-86.

Duncan, S. F., Steed, A., \& Needham, C. M. (2009). A comparison evaluation study of web-based and traditional marriage and relationship education. Journal of Couple \& Relationship Therapy, $8(2), 162-180$.

Loew, B., Rhoades, G., Markman, H., Stanley, S., Pacifici, C., White, L., \& Delaney, R. (2012). Internet delivery of PREP-based relationship education for at-risk couples. Journal of Couple \& Relationship Therapy, 11(4), 291-309. 
Roddy, M. K., Nowlan, K. M., \& Doss, B. D. (2017). A randomized controlled trial of coach contact during a brief online intervention for distressed couples. Family Process, 56(4), 835-851.

Roddy, M. K., Rothman, K., \& Doss, B. D. (2018). A randomized controlled trial of different levels of coach support in an online intervention for relationship distress. Behaviour Research and Therapy, 110, 47-54.

Roddy, M. K., Rhoades, G. K., \& Doss, B. D. (2020). Effects of ePREP and OurRelationship on low-income couples' mental health and health behaviors: A randomized controlled trial. Prevention Science. https://doi.org/10.1007/s11121-020-01100-y
Roddy, M. K., Knopp, K., Georgia Salivar, E., \& Doss, B. D. (2020b). Maintenance of relationship and individual functioning gains following online relationship programs for low-income couples. Family Process. https://doi.org/10.1111/famp.12541

Publisher's Note Springer Nature remains neutral with regard to jurisdictional claims in published maps and institutional affiliations. 